\title{
ON THE CONNECTEDNESS OF HOMOMORPHISMS IN TOPOLOGICAL DYNAMICS
}

\author{
BY
}

\author{
D. McMAHON AND T. S. WU
}

ABSTRACT. Let $(X, T)$ be a minimal transformation group with compact Hausdorff phase space. We show that if $\phi:(X, T) \rightarrow(Y, T)$ is a distal homomorphism and has a structure similar to the structure Furstenberg derived for distal minimal sets, then for $T$ belonging to a class of topological groups $T$, the homomorphism $X \rightarrow X / S(\phi)$ has connected fibers, where $S(\phi)$ is the relativized equicontinuous structure relation. The class $T$ is defined by Sacker and Sell as consisting of all groups $T$ with the property that there is a compact set $K \subseteq T$ such that $T$ is generated by each open neighborhood of $K$. They show that for such $T$, a distal minimal set which is a finite-toone extension of an almost periodic minimal set is itself an almost periodic minimal set. We provide an example that shows that the restriction on $T$ cannot be dropped.

As one of the preliminaries to the above we show that given $\phi:(X, T)$ $\rightarrow(Y, T)$, the relation $R c(\phi)$ induced by the components in the fibers relative to $\phi$, i.e., $\left(x, x^{\prime}\right) \in R c(\phi)$ if and only if $x$ and $x^{\prime}$ are in the same component of $\phi^{-1}(\phi(x))$, is a closed invariant equivalence relation.

We also consider the question of when a minimal set $(X, T)$ is such that $Q(x)$ is finite for some $x$ in $X$, where $Q$ is the regionally proximal relation. This problem was motivated by Veech's work on almost automorphic minimal sets, i.e., the case in which $Q(x)$ is a singleton for some $x$ in $X$.

Introduction. Let $(X, T)$ be a minimal transformation group with compact Hausdorff phase space. In his paper on distal transformation groups, Ellis proved that a distal minimal set $(X, T)$ is equicontinuous if $X$ is totally disconnected and $T$ is generative, i.e., $T$ is an abelian group generated by some compact neighborhood of the identity. In this paper we generalize this result by requiring that $T$ be the direct product of a compact group with a compactly generated separable group rather than that $T$ be generative. More generally, we show that if $\phi:(X, T) \rightarrow(Y, T)$ is a distal homomorphism and has a structure similar to the structure Furstenberg derived for distal minimal sets (see Definition 3.5), then for $T$ belonging to a class of topological groups $T$, the homomorphism

Presented to the Society, January 23, 1975; received by the editors July 5, 1974 and, in revised form, March 19, 1975.

AMS (MOS) subject classifications (1970). Primary 54H20.

Key words and phrases. Minimal, transformation group homomorphism, relativized equicontinuous structure relation, connected. 
$X \rightarrow X / S(\phi)$ has connected fibers, where $S(\phi)$ is the relativized equicontinuous structure relation. The class $T$ is defined by Sacker and Sell in [11] as consisting of all groups $T$ with the property that there is a compact set $K \subseteq T$ such that $T$ is generated by each open neighborhood of $K$. They show that for such $T$, a distal minimal set which is a finite-to-one extension of an almost periodic minimal set is itself an almost periodic minimal set. We provide an example that shows that the restriction on $T$ cannot be dropped.

The above is done in $\S 3$, and was motivated in part by Ellis's paper on the construction of minimal discrete flows, in which given a minimal set $(Y, T)$ with $T$ the integers, an extension $(X, T)$ of $(Y, T)$ is constructed and the examples provided all have homomorphisms $\phi: X \rightarrow Y$ with connected fibers.

$\S 2$ consists of some preliminaries to $\S 3$, that are felt to be of interest in themselves. In particular we show that given $\phi:(X, T) \rightarrow(Y, T)$, the relation $R c(\phi)$ induced by the components in the fibers relative to $\phi$, i.e., $\left(x, x^{\prime}\right) \in R c(\phi)$ if and only if $x$ and $x^{\prime}$ are in the same component of $\phi^{-1}(\phi(x))$, is a closed invariant equivalence relation.

In $\$ 1$ we give a very direct proof of the result of Ellis that a minimal set $(X, T)$ that is an almost periodic extension of a minimal set $(Y, T)$ is the homomorphic image of a compact group extension of $(Y, T)$.

In $\S 4$ we consider the question of when a minimal set $(X, T)$ is such that $Q(x)$ is finite for some $x$ in $X$, where $Q$ is the regionally proximal relation. This problem was motivated by Veech's work on almost automorphic minimal sets, i.e., the case in which $Q(x)$ is a singleton for some $x$ in $X$.

Definitions and notation. $(X, T)$ will denote a transformation group with compact Hausdorff phase space. All homomorphisms will be surjective. An extension $\phi:(X, T) \rightarrow(Y, T)$ will be said to be a finite extension and the homomorphism $\phi$ will be said to be finite-to-one or to have finite fibers if $\phi^{-1}(y)$ is finite for every $y$ in $Y$. The extension and the homomorphism are said to have connected fibers if $\phi^{-1}(y)$ is connected for every $y \in Y$.

$T$ will denote the class of all groups $T$ with the property that there is a compact set $K \subseteq T$ such that $T$ is generated by any open neighborhood of $K$. This class includes the class of compactly generated groups.

Suppose $\phi:(X, T) \rightarrow(Y, T)$. Let $R(\phi)=\left\{\left(x, x^{\prime}\right): \phi(x)=\phi\left(x^{\prime}\right)\right\}$. Then $P(\phi)=\left\{\left(x, x^{\prime}\right) \in R(\phi)\right.$ : there is a net $t_{n}$ in $T$ with $\left.\lim x t_{n}=\lim x^{\prime} t_{n}\right\}$ is the relativized proximal relation; $Q(\phi)=\left\{\left(x, x^{\prime}\right) \in R(\phi)\right.$ : there exist nets $t_{n}$ in $T$ and $\left(x_{n}, x_{n}^{\prime}\right)$ in $R(\phi)$ such that $\left(x_{n}, x_{n}^{\prime}\right) \rightarrow\left(x, x^{\prime}\right)$ and $\left.\lim x t_{n}=\lim x^{\prime} t_{n}\right\}$ is the relativized regionally proximal relation. $S(\phi)$ will denote the relativized equicontinuous structure relation and is the smallest closed invariant equivalence relation containing $Q(\phi)$. $\phi$ is an almost periodic homomorphism if and only if 
$Q(\phi)$ equals the diagonal of $X \times X$. When $Y$ is a singleton, we will use the notation $P$ or $P_{X}, Q$ or $Q_{X}, S$ or $S_{X}$.

A bitransformation group $(K, X, T)$ consists of a transformation group $(X, T)$ and a transformation group $(K, T)$ such that $(k x) t=k(x t)$ for all $k \in K$, $x \in X, t \in T$. Due to this last condition if $K$ is compact the orbit space of $K$ forms a transformation group, more specifically, $(X / K, T)$ is a transformation group where $X / K$ is obtained by identifying $x$ and $x^{\prime}$ if $x^{\prime} \in K x$.

\section{$\S 1$}

In this section we give a very direct proof of a result of Ellis (see 12.12, 12.13, and 14.26 of [6]). The proof in [6] depends on the algebraic theory and $\tau$-topology of Ellis. We provide an elementary proof here. Persons familiar with 12.12 and 12.13 may wish to skim the first three paragraphs of the proof which develop ideas similar to 12.12 and 12.13 in much the same manner.

For this section let $(M, T)$ be a minimal right ideal in the enveloping semigroup $E$ of a minimal set $(Z, T) . E$ is the closure of the transition group of $(Z, T)$ in $Z^{Z}$, where $Z^{Z}$ is the collection of functions from $Z$ to $Z$ topologized by the pointwise convergence topology. With composition of functions as binary operation, $Z^{Z}$ is a compact semigroup and its properties are developed in [2], [6]. Fix an idempotent $u$ in $M$ and let $G=M u$; then $G$ is a group. Furthermore, $M=G J$ where $J$ is the set of idempotents in $M$. Suppose $(X, T)$ is a minimal set and $\psi$ is a homomorphism of $(M, T)$ onto $(X, T)$. For $p$ in $M$ and $x$ in $X$ we define $x p=\psi(q p)$ if $\psi(q)=x$; this definition is independent of the $q$ taken with $\psi(q)=x$ and can also be defined as $x p=\lim x t_{n}$ where $t_{n}$ is a net in $T$ that converges to $p$ when considered as a net in the transition group of $(Z, T)$ and thus as a subset of $E$. Let $x_{0}=\psi(u)$ and $G(\psi)=\psi^{-1}\left(x_{0}\right) \cap G=$ $\left\{g \in G: x_{0} g=x_{0}\right\}$. Note that $u \in G(\psi), G(\psi)$ is a subgroup of $G$, and for any net $g_{n}$ in $G(\psi)$ with limit $p$ in $M, \psi(p)=x_{0}$ and $\psi(p u)=x_{0} u=x_{0}$, thus $p u \in$ $G(\psi)$. Then $\left\{p: x_{0} p=x_{0}\right\}=G(\psi) J^{\prime}$ where $J^{\prime}=\left\{v \in J: x_{0} v=x_{0}\right\}$. Also for any $x$ in $X, \psi^{-1}(x)=G(\psi) g J(x)$ where $g \in G$ with $x u=x_{0} g$ and $J(x)=\{v \in J$ : $x v=x\}$ and $\{p \in M: x p=x\}=g^{-1} G(\psi) g J(x)$.

A homomorphism $\phi:(X, T) \rightarrow(Y, T)$ is called distal if $P(\phi)=\Delta$ (the diagonal of $X \times X)$ and is called almost periodic if $Q(\phi)=\Delta$. Note that if $\phi$ is almost periodic, then $T$ acts equicontinuously on each fiber of $\phi$.

(1.1) Proposition. Given a distal homomorphism $\phi$ from a minimal set $(X, T)$ onto a minimal set $(Y, T)$, there exists a bitransformation group $(K, W, T)$ and a subgroup $L$ of $K$ such that $(W, T)$ is minimal, $K$ acts freely on $W$, and $(W / L, T)$ can be identified with $(X, T),(W / K, T)$ can be identified with $(Y, T)$ and the natural projection of $W / L$ onto $W / K$ can be identified with $\phi$. If $\phi$ is an almost periodic homomorphism, then $K$ and $L$ may be taken to be a compact 
Hausdorff topological group and the fibers of $\phi$ are homeomorphic to the coset space $K / L=\{k L: k \in K\}$.

Proof. Fix $x_{0}$ in $X$ and let $\phi\left(x_{0}\right)=y_{0}$. Let $(M, T)$ be a minimal right ideal in the enveloping semigroup of $(X, T)$ and let $J$ be the set of idempotents in $M$. Fix an element $u$ in $J$ such that $x_{0} u=x_{0}$ and let $G=M u$. Then $\{p \in M$ : $\left.y_{0} p=y_{0}\right\}=F J\left(y_{0}\right)$ where $F=\left\{f \in G: y_{0} f=y_{0}\right\}$ and $J\left(y_{0}\right)=\left\{v \in J: y_{0} v=y_{0}\right\}$; similarly $\left\{p \in M: x_{0} p=x_{0}\right\}$ is the product of a subset $A$ of $G$ with a subset $J\left(x_{0}\right)$ of $J$. Note that $J\left(y_{0}\right)=J\left(x_{0}\right)$ since $\phi$ is distal. Consider the transformation group $\left(\Pi\left\{X_{f}: f \in F\right\}, T\right)$ where $X_{f}$ is a copy of $X$ indexed by $f$. Let $w_{0}=$ $\left\langle x_{0} f\right\rangle$ be the element of the product with $f$-coordinate $x_{0} f$ and let $W$ be the orbit closure of $w_{0}$. (Note this is similar to a technique of Veech in $[14$, p. 36].) Then $(W, T)$ is minimal and the projection $p$ of $W$ onto its $u$ th factor is a homomorphism of $(W, T)$ onto $(X, T)$ with $p\left(w_{0}\right)=x_{0}$. Note that $(W, T)$ is a homomorphic image of $(M, T)$ and that $u \in A \subseteq F \subseteq G$. Let $\left\{p \in M: w_{0} p=w_{0}\right\}=$ $B J\left(w_{0}\right) \subseteq G J$. Note that for $g$ in $G, w_{0} g=w_{0}$ iff $x_{0} f g=x_{0} f$ for every $f$ in $F$ iff $x_{0} f g f^{-1}=x_{0}$ for every $f$ in $F$ iff $f g f^{-1} \in A$ for every $f$ in $F$ iff $g \in f^{-1} A f$ for every $f$ in $F$ iff $g \in \bigcap\left\{f^{-1} A f: f \in F\right\}$. So $B=\bigcap\left\{f^{-1} A f: f \in F\right\}$ and thus is a normal subgroup of $F$. Also $J\left(w_{0}\right)=J\left(y_{0}\right)$ since if $v \in J\left(y_{0}\right)$, then for every $f$ in $F, \phi\left(x_{0} f\right)=y_{0} f=y_{0}$, so $J\left(x_{0} f\right)=J\left(y_{0}\right)$ since $\phi$ is distal, so $x_{0} f v=x_{0} f$, and thus $w_{0} v=w_{0}$ and $J\left(w_{0}\right) \supseteq J\left(y_{0}\right)$; the other containment is clear. This equality implies that the homomorphism $\theta=\phi \circ p$ is a distal homomorphism of $(W, T)$ onto $(Y, T)$.

For each $f$ in $F$ define a homomorphism $f^{*}:(W, T) \rightarrow(W, T)$ by $f^{*}\left(w_{0} g v\right)=w_{0} f g v$ where $g v \in M=G J, g \in G, v \in J$. This is well defined since if $w_{0} g v=w_{0} g^{\prime} v^{\prime}$, then $x_{0} f_{1} g v=x_{0} f_{1} g^{\prime} v^{\prime}$ for every $f_{1}$ in $F$ and noting that $f_{1} f \in$ $F$, we see that $x_{0} f_{1} f g v=x_{0} f_{1} f g^{\prime} v^{\prime}$ for every $f_{1}$ in $F$ and $w_{0} f g v=w_{0} f g^{\prime} v^{\prime}$. Then clearly the map $f \mapsto f^{*}$ is a group homomorphism from $F$ into the group of homeomorphisms of $W$ and its kernel is $B$. If we identify $F / B$ with its image as a subgroup of the group of homeomorphisms of $W$ and topologize it with the compact-open topology, then it is a Hausdorff topological group and $(F / B, W, T)$ is a bitransformation group.

Let $K=F / B$ and $L=A / B$. Now $w^{\prime} \in K w$ iff $w^{\prime}=w_{0} f g v$ for some $f \in F$, where $w=w_{0} g v$, iff $\theta\left(w^{\prime}\right)=y_{0} f g v=y_{0} g v=\theta(w)$; so $(W / K, T) \approx(Y, T)$. Also $K$ acts freely on each fiber of $\theta$ since the action is determined by its effect on $w_{0}$ and $\theta$ is distal. Similarly $w^{\prime} \in L w$ iff $p(w)=p\left(w^{\prime}\right) ;(W / L, T) \approx(X, T)$; and $L$ acts freely on each fiber of $p$. It is clear that we may identify $\phi$ with the natural homomorphism of $W / L$ onto $W / K$ that sends $L w$ to $K w$. Note then that the fibers of $\phi$ are one-to-one continuous images of $K / L=\{k L: k \in K\}$.

We wish to show that if $\phi$ is almost periodic, then $F / B$ with the compactopen topology is a compact Hausdorff topological group. First we show that if 
$\phi$ is almost periodic, then so is $\theta=\phi^{\circ} p$. Suppose $\left(z, z^{\prime}\right) \in Q(\theta)$. If $p_{f}$ is the projection of $\pi\left\{X_{f}: f \in F\right\}$ onto the $f$-factor $X_{f}$, we note that $\phi \circ p_{f}=\theta=\phi \circ p$. So $\left(p_{f}(z), p_{f}\left(z^{\prime}\right)\right) \in Q(\phi)$ and then $p_{f}(z)=p_{f}\left(z^{\prime}\right)$ since $Q(\phi)$ equals the diagonal of $X \times X$. This holds for every $f \in F$ and so $z=z^{\prime}$. Thus $\theta$ is almost periodic.

Furthermore, $F / B$ is equicontinuous. Indeed if not, then there exist nets $\left(w_{n}, w_{n}^{\prime}\right) \in R(\theta)$ and $f_{n}$ in $F$ such that $\left(w_{n}, w_{n}^{\prime}\right) \rightarrow(\hat{w}, \hat{w})$ and $f_{n}^{*}\left(w_{n}, w_{n}^{\prime}\right)$ $\rightarrow\left(w, w^{\prime}\right)$ for some $\hat{w}, w, w^{\prime}$ in $W$, with $w \neq w^{\prime}$. Note that $\theta(\hat{w})=\theta(w)=$ $\theta\left(w^{\prime}\right)$. Then $w_{n}=w_{0} g_{n} v_{n}$ and $w_{n}^{\prime}=w_{0} f_{n}^{\prime} g_{n} v_{n}$ for some $v_{n} \in J, g_{n} \in G, f_{n}^{\prime} \in$ $F$ and $w=f^{*} \hat{w}$ for some $f \in F$. We have then $w_{0} f g_{n} v_{n}=f^{*}\left(w_{0} g_{n} v_{n}\right) \rightarrow$ $f^{*}(\hat{w})=w, w_{0} f f_{n}^{\prime} g_{n} v_{n}=f^{*}\left(w_{0} f_{n}^{\prime} g_{n} v_{n}\right) \rightarrow f^{*}(\hat{w})=w, w_{0} f_{n} g_{n} v_{n} \rightarrow w$, and $w_{0} f_{n} f_{n}^{\prime} g_{n} v_{n} \rightarrow w^{\prime}$. So $\left(w_{0} f, w_{0} f_{n}\right) g_{n} v_{n} \rightarrow(w, w)$ and $\left(w_{0} f, w_{0} f_{n}\right) f_{n}^{\prime} g_{n} v_{n}$ $\rightarrow\left(w, w^{\prime}\right)$. Then by multiplying $\left(w_{0} f, w_{0} f_{n}\right) f_{n}^{\prime} g_{n} v_{n}$ on the right by $g_{n}^{-1}\left(f_{n}^{\prime}\right)^{-1} g_{n} v_{n}$ it is clear that $\left(w, w^{\prime}\right) \in Q(\theta)$ and so $w=w^{\prime}$. This gives a contradiction and so $F / B$ acts equicontinuously.

By Ascoli's theorem $K=F / B$ is relatively compact. Suppose $k_{n}$ is a net in $K$ converging to a homeomorphism $c$ of $W$. We wish to show that $c \in K$. Now $c\left(w_{0}\right)=\lim k_{n} w_{0}=w_{0} f$ for some $f \in F$. Then $c\left(w_{0} u t\right)=w_{0}$ fut for $t \in T$ and since $(M, T)$ is minimal and $c$ is continuous, $c\left(w_{0} g v\right)=w_{0} f g v$ for $g v \in M$; thus $c=f^{*} \in F / B$. So $K$ is compact. Clearly $L$ is also compact. The compactness of $K$ implies that the fibers of $\phi$ are homeomorphic to the coset space $K / L=\{k L: k \in K\}$.

(2.1) Proposition. Suppose $(X, T)$ is minimal, $\phi:(X, T) \rightarrow(Y, T)$ is an almost periodic homomorphism and $\psi:(Y, T) \rightarrow(Z, T)$ is a finite-to-one open homomorphism. Then $\alpha=\psi \circ \phi: X \rightarrow Z$ is an almost periodic homomorphism.

Proof. First note that $\psi$ is a distal homomorphism. We will show that $Q(\alpha)$ equals the diagonal of $X \times X$ given that $P(\psi)$ equals the diagonal of $Y \times Y$ and $Q(\phi)$ equals the diagonal of $X \times X$.

Let $\left(x, x^{\prime}\right) \in Q(\alpha)$. Then since $\phi$ is open, there exist nets $x_{n}, x_{n}^{\prime}$ in $\phi^{-1}(\phi(x))$ and $s_{n}, t_{n}$ in $T$ such that $\left(x_{n}, x_{n}^{\prime}\right) s_{n} \rightarrow\left(x, x^{\prime}\right)$ and $\left(x_{n}, x_{n}^{\prime}\right) t_{n} \rightarrow$ $(\hat{x}, \hat{x})$, for some $\hat{x} \in X$. Then $\left(\phi\left(x_{n}\right), \phi\left(x_{n}^{\prime}\right)\right) t_{n} \rightarrow(\phi(\hat{x}), \phi(\hat{x}))$. Now since $\phi^{-1}(\phi(x))$ is finite, we may assume that $\phi\left(x_{n}\right)$ and $\phi\left(x_{n}^{\prime}\right)$ are fixed elements of $Y$, i.e., $\phi\left(x_{n}\right)=y, \phi\left(x_{n}^{\prime}\right)=y^{\prime}$ for some $y, y^{\prime} \in Y$ and all $n$ (otherwise take subnets). Then by the above $\left(y, y^{\prime}\right) t_{n} \rightarrow(\phi(\hat{x}), \phi(\hat{x}))$ and so $\left(y, y^{\prime}\right) \in P(\psi)$. Thus $y=y^{\prime}$, which in turn implies that $\phi\left(x_{n}\right)=y=y^{\prime}=\phi\left(x_{n}^{\prime}\right)$, so $\left(x_{n}, x_{n}^{\prime}\right) \in R(\phi)$ and $\left(x, x^{\prime}\right) \in Q(\phi)$. Thus $x=x^{\prime}$ and so $Q(\alpha)$ equals the diagonal of $X \times X$.

(2.2) Definition. Given a homomorphism $\phi$ of $(X, T)$ onto $(Y, T)$, let $R c(\phi)$ be the relation induced by the components in the fibers relative to $\phi$; 
that is, $\left(x, x^{\prime}\right) \in R c(\phi)$ if and only if $x$ and $x^{\prime}$ are in the same component of $\phi^{-1}(\phi(x))$.

It is clear that $R c(\phi)$ is a $T$-invariant equivalence relation. In fact, it is also closed as the following proposition shows.

(2.3) Proposition. $R c(\phi)$ is closed.

Proof. Let $\left(x_{n}, x_{n}^{\prime}\right)$ be a net in $R c(\phi)$ such that $\lim \left(x_{n}, x_{n}^{\prime}\right)=\left(x, x^{\prime}\right)$. If $\left(x, x^{\prime}\right) \notin R c(\phi)$, then there exist disjoint closed subsets $A$ and $B$ of $\phi^{-1}(\phi(x))$ with $x \in A, x^{\prime} \in B$ and $A \cup B=\phi^{-1}(\phi(x))$. There exist disjoint open sets $U$ and $V$ in $X$ such that $A \subseteq U$ and $B \subseteq V$. Since $\phi$ is a closed map, there is an open neighborhood $W$ of $\phi(x)$ in $Y$ such that $\phi^{-1}(W) \subseteq U \cup V$. Now for some $n, x_{n} \in U, x_{n}^{\prime} \in V$ and $\phi\left(x_{n}\right)=\phi\left(x_{n}^{\prime}\right) \in W$. So $\phi^{-1}\left(\phi\left(x_{n}\right)\right) \subseteq U \cup V$ and $\left\{U \cap \phi^{-1}\left(\phi\left(x_{n}\right)\right), V \cap \phi^{-1}\left(\phi\left(x_{n}\right)\right)\right\}$ gives a separation of $\phi^{-1}\left(\phi\left(x_{n}\right)\right)$. This is a contradiction.

Now consider the following commutative diagram, with $\theta, \phi$ onto homomorphisms and $\alpha=\theta \circ \phi$. We note that for $z \in Z$, if $C$ is a component of $\alpha^{-1}(z)$, then $\phi(C)$ is contained in a component of $\theta^{-1}(z)$. Thus $\phi$ induces canonically a homomorphism $\phi_{c}$ of $X / R c(\alpha)$ onto $Y / R c(\theta)$.

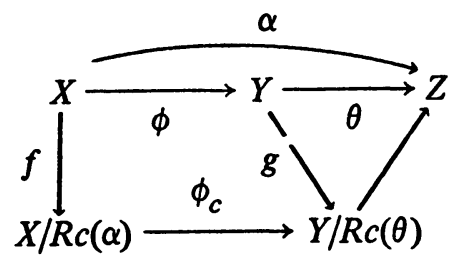

(2.4) LEMMA. If $\phi$ is open and $\left(y, y^{\prime}\right) \in R c(\theta)$, then for every $x$ in $X$ with $\phi(x)=y$ there exists $x^{\prime}$ in $X$ with $\phi\left(x^{\prime}\right)=y^{\prime}$ and $\left(x, x^{\prime}\right) \in R c(\alpha)$.

Proof. We note $\phi \mid \alpha^{-1}(z): \alpha^{-1}(z)=\phi^{-1}\left(\theta^{-1}(z)\right) \rightarrow \theta^{-1}(z)$ is an open map. Therefore $\phi$ maps components of $\alpha^{-1}(z)$ onto components of $\theta^{-1}(z)$ and the conclusion of the lemma follows.

(2.5) Proposition. If $\phi$ is a distal homomorphism, so is $\phi_{c}$.

Proof. We note that since $\phi$ is distal, it is open. Suppose $x, x^{\prime}$ are elements of $X$ such that $\phi_{c}(f(x))=\phi_{c}\left(f\left(x^{\prime}\right)\right)$, we will show that $f(x)$ and $f\left(x^{\prime}\right)$ are distal.

Note first that $g(\phi(x))=\phi_{c}(f(x))=\phi_{c}\left(f\left(x^{\prime}\right)\right)=g\left(\phi\left(x^{\prime}\right)\right)$ and so $\left(\phi(x), \phi\left(x^{\prime}\right)\right)$ $\in R c(\theta)$. By 1.4 there exists $x_{1}$ in $X$ such that $\left(x, x_{1}\right) \in R c(\alpha)$ and $\phi\left(x_{1}\right)=$ $\phi\left(x^{\prime}\right)$. Since $\phi$ is a distal homomorphism, $x_{1}$ and $x^{\prime}$ are distal and therefore $f\left(x_{1}\right)$ and $f\left(x^{\prime}\right)$ are distal. The proof is complete since $f\left(x_{1}\right)=f(x)$.

(2.6) Proposition. If $(X, T)$ is minimal and $\phi$ is an almost periodic homomorphism from $(X, T)$ onto $(Y, T)$ with totally disconnected fibers, then 
$(X, T)$ is isomorphic to the inverse limit of finite extensions $\left(X_{N}, T\right)$ of $(Y, T)$ such that the connecting homomorphisms $\phi_{N}^{N^{\prime}}:\left(X_{N}, T\right) \rightarrow\left(X_{N^{\prime}}, T\right)$ for $N^{\prime} \leqslant$ $N$, are induced by $\phi$.

Proof. By 1.1 there is a bitransformation group $(K, W, T)$ with $K$ a compact Hausdorff topological group acting freely on $W$ and there is a compact subgroup $L$ of $K$ such that we may identify $(W / K, T)$ with $(Y, T),(W / L, T)$ with $(X, T)$, and the projection of $(W / L, T)$ onto $(W / K, T)$ with the homomorphism $\phi:(X, T) \rightarrow(Y, T)$.

Let $N$ be an open-closed normal subgroup of $K$ and let $\left(X_{N}, T\right)=(W / N L, T)$. Order the set of open-closed normal subgroups of $K$ by containment $\left(N^{\prime} \leqslant N\right.$ if $\left.N^{\prime} \supseteq N\right)$. The connecting homomorphism $\phi_{N}^{N^{\prime}}$ is the projection of $X / N L$ onto $X / N^{\prime} L$. Note that $X_{N}$ is a finite extension of $Y$ since the fibers of the projection of $W / N L$ onto $W / K$ are homeomorphic to $K / N L$ which is finite since $N L$ is an open subgroup and $K$ is compact.

To show that $W / L$ is the inverse limit of $\left\{X_{N}, \phi_{N}^{N^{\prime}}\right\}$ for all open-closed normal subgroups $N, N^{\prime}$ of $K$, we need to show that if $x, y \in W$ with $y \notin L x$, that is, if $x$ and $y$ have distinct images in $W / L$, then there exists an open-closed normal subgroup $N$ of $K$ such that $y \notin N L x$. If $y \notin K x$, let $N=K$. If $y \in K x$, then since $K / L$ is homeomorphic to the fibers of $\phi$ which are totally disconnected, the connected component $C_{0}$ of $K$ is contained in $L$. Since $L L=L$ and $L$ is compact, there exists an open neighborhood $V$ of $L$ such that $y \notin V L x$. Then since $C_{0} \subseteq L \subseteq V L$ is the intersection of open-closed sets, there exists an open-closed set $V^{\prime} \subseteq V L$. By Theorem 7.6 of [9] there exists an open-closed normal subgroup $N$ of $K$ such that $N \subseteq V^{\prime}$. Then $N L \subseteq V^{\prime} L \subseteq V L L=V L$ and $y \notin N L x$.

(2.7) COROllary. If $(X, T)$ is minimal and $\phi$ is an almost periodic homomorphism of $(X, T)$ onto $(Y, T)$, then $\phi_{c}$ is an almost periodic homomorphism.

Proof. By the above $X / R c(\phi)$ is the inverse limit of finite extensions. Let $\beta$ be the homomorphism from $X / R c(\phi)$ onto $Y$ induced by $\phi$. By the fact that the inverse limit over a directed set of compact connected sets is connected, one can show that $(X / R c(\phi)) / R c(\theta \circ \beta)$ is the inverse limit of finite extensions of $Y / R c(\theta)$. Note that $X / R c(\theta \circ \phi)$ is isomorphic to $(X / R c(\phi)) / R c(\theta \circ \beta)$ and that by 2.1 and $2.5, X / R c(\theta \circ \phi)$ is an almost periodic extension.

\section{$\S 3$}

As in [11], define $T$ to be the class of all Hausdorff topological groups $T$ that contain a compact set $K$ such that $T$ is generated by each neighborhood of $K$. The discussion and 3.1 below are condensed from [11].

If $\phi: W \rightarrow Y$ and $0<N<\infty$ then $W$ is said to be an $N$-fold covering of $Y$ with covering projection $\phi$ if $\operatorname{card} \phi^{-1}(y)=N$ for all $y \in Y$ and for each 
$y \in Y$ there is an open neighborhood $V$ of $y$ such that $\phi^{-1}(V)$ consists of $N$ disjoint open sets $U_{i}$ and $\left(\phi \mid U_{i}\right): U_{i} \rightarrow V$ is a homeomorphism, $i=1, \ldots, N$.

(3.1) Theorem (Structure Theorem). Let $(W, T, \pi)$ and $(Y, T, \sigma)$ be transformation groups where $W$ and $Y$ are compact Hausdorff spaces. Assume $(Y, T, \sigma)$ is minimal and $\phi: W \rightarrow Y$ a homomorphism. Then the following statements are equivalent:

(A) $\phi$ is distal and for some $y_{0} \in Y$, card $\phi^{-1}\left(y_{0}\right)=N, 0<N<\infty$.

(B) $W$ is an $N$-fold covering of $Y$.

Finally if any of these hold then $W$ can be expressed as the disjoint union $W=W_{1} \cup \ldots \cup W_{k}$ of compact minimal sets, where each $W_{i}$ is an $n_{i}$-fold covering of $Y$ and $n_{1}+\ldots+n_{k}=N$.

Proof. Theorem 1 of [11].

(3.2) Proposition. Given transformation groups $(X, T),(Y, T),(Z, T)$ and homomorphism $\phi:(X, T) \rightarrow(Y, T), \psi:(Y, T) \rightarrow(Z, T)$ such that $X$ is an $N$-fold covering of $Y$ with covering map $\phi$, and $\psi$ is an almost periodic homomorphism, if $T \in T$, then $\psi \circ \phi$ is an almost periodic homomorphism.

The above proposition is a generalization of a result of Sacker and Sell [11]. Their result is obtained when $Z$ is taken to be a singleton in the above proposition. The proof requires only minor changes in their proof. We will give a proof of 3.2 to indicate these changes and the role $T$ plays. We start with a lemma that is purely topological.

(3.3) LEMMA. Let $X$ be an $N$-fold covering of $Y$ with covering map $\phi$, and let $X$ and $Y$ be compact Hausdorff. Then there exists an open index $\alpha$ on $X$ such that for every index $\gamma$ on $X$ there is an index $\beta$ on $Y$ with the property that $\left(\phi(x), \phi\left(x^{\prime}\right)\right) \in \beta$ implies that $\left(x, x^{\prime}\right) \notin \alpha$ or $\left(x, x^{\prime}\right) \in \gamma$.

Proof. For each $y$ in $Y$, let $V_{y}$ be a neighborhood of $y$ with $\phi^{-1}(y)$ a disjoint union of $N$ open sets $W_{y}^{1}, \ldots, W_{y}^{N}$ each of which is homeomorphic to $V_{y}$ under the restriction of $\phi$. Let $\phi^{-1}(y)=\left\{x_{y}^{1}, \ldots, x_{y}^{N}\right\}, x_{y}^{i} \in W_{y}^{i}$; then there exists an open index $\alpha_{y}$ on $X$ with $\alpha_{y}\left(x_{y}^{i}\right) \subseteq w_{y}^{i}$ for $i=1, \ldots, N$. Let $\left.U_{y}=\bigcap\left\{\alpha_{y}\left(x_{y}^{i}\right)\right): i=1, \ldots, N\right\}$. The collection of $U_{y}$ for $y$ in $Y$ is an open cover of $Y$, so we may take a finite subcover $U_{y_{1}}, \ldots, U_{y_{m}}$. Let $\alpha=$ $\bigcap\left\{\alpha_{y_{i}}: i=1, \ldots, m\right\}$. There exists an index $\delta$ on $Y$ such that $\left(y, y^{\prime}\right) \in \delta$ implies $y$ and $y^{\prime}$ are in $U_{y_{i}}$ for some $i$. Now given an index $\gamma$ on $X$, let $\beta=\delta$ $\cap(\phi \times \phi)(\gamma)$. Then $\left(\phi(x), \phi\left(x^{\prime}\right)\right) \in \beta$ implies $\phi(x)$ and $\phi\left(x^{\prime}\right)$ are in $U_{y_{i}}$ for some $i$. This implies $x \in \alpha_{y_{i}}\left(x_{y_{i}}^{j}\right)$ for some $j$, and $x^{\prime} \in \alpha_{y_{i}}\left(x_{y_{i}}^{k}\right)$ for some $k$. Recalling that $\alpha_{y_{i}}\left(x_{y_{i}}^{j}\right) \subseteq w_{y_{i}}^{j}$ we see that if $j \neq k$, then $\left(x, x^{\prime}\right) \notin \alpha$, and if $j=k$, then (since $\left.\phi\right|_{w_{y_{i}}^{j}}$ is one-to-one) one can verify that $\left(x, x^{\prime}\right) \in \gamma$. 
Proof OF 3.2. We wish to show that given an index $\gamma$ on $X$ there exists an index $\mu$ on $X$ such that $(\mu \cap R(\phi \circ \psi)) T \subseteq \gamma$.

Let $\alpha$ be as in the above lemma and let $K$ be a compact subset of $T$ such that $T$ is generated by each neighborhood of $K$. Note that $\Delta K=\Delta$ (= the diagonal of $X$ ), so $\delta V \subseteq \alpha$ for some index $\delta$ on $X$ and some set $V$ that generates $T$. (This is the sole use of the condition $T \in T$.) We may assume that $\gamma \subseteq \delta$. Take $\beta$ as in the lemma so that $\left(\phi(x), \phi\left(x^{\prime}\right)\right) \in \beta$ implies $\left(x, x^{\prime}\right) \in \gamma$ or $\left(x, x^{\prime}\right) \notin \alpha$.

By the equicontinuity of $(Y, T)$, there is an index $\nu$ on $Y$ such that $(\nu \cap R(\psi)) T \subseteq \beta$. Now let $\mu=\gamma \cap(\phi \times \phi)^{-1}(\nu)$. Suppose $\left(x, x^{\prime}\right) \in \mu \cap$ $R(\phi \circ \psi)$ and $t \in T$, we wish to show that $\left(x, x^{\prime}\right) t \in \gamma$. Now $t=v_{0} v_{1} \ldots v_{n}$ where $v_{0}=$ identity and $v_{i} \in V$ for $i=1, \ldots, n$. If $\left(x, x^{\prime}\right) t \notin \gamma$, then for some $i,\left(x, x^{\prime}\right) v_{0} \ldots v_{i} \in \gamma$ and $\left(x, x^{\prime}\right) v_{0} \ldots v_{i} v_{i+1} \notin \gamma$. Note that $\left(\left(x, x^{\prime}\right) v_{0} \ldots v_{i}\right) v_{i+1} \in \alpha$ since $\gamma \subseteq \delta$. However $\left(\phi(x), \phi\left(x^{\prime}\right)\right) \in \nu \cap R(\psi)$, so $\left(\phi(x), \phi\left(x^{\prime}\right)\right) v_{0} \cdots v_{i} v_{i+1} \in \beta$, and thus $\left(x, x^{\prime}\right) v_{0} \cdots v_{i} v_{i+1} \in \gamma$ or $\left(x, x^{\prime}\right) v_{0}$ $\cdots v_{i} v_{i+1} \notin \alpha$. This gives a contradiction and the proposition follows.

(3.4) ExAmple 1. The following is an example of a finite distal extension of an equicontinuous minimal set which is not equicontinuous and so shows the need for some restrictions on $T$ in the above.

Let $(X, T)$ be an equicontinuous minimal set with $X$ totally disconnected, fix $x^{*}$, and let $V^{i}$ be a filter of open-closed sets converging to $x^{*}$ with $x^{*} \notin V^{i}$. Take two copies of $X$ and denote the topological sum of these two copies by $X_{1} \cup X_{-1}=W$. Define $b_{i}: W \rightarrow W$ by

$$
x_{\epsilon} b_{i}=\left\{\begin{array}{ll}
x_{\epsilon} & \text { if } x_{\epsilon} \notin V_{\epsilon}^{i} \\
x_{-\epsilon} & \text { if } x_{\epsilon} \in V_{\epsilon}^{i}
\end{array} \quad \text { and for } t \in T\right.
$$

define $x_{\epsilon} t=(x t)_{\epsilon}$; then $\left(W, T^{\prime}\right)$, where $T^{\prime}$ is the group generated by $T$ and $\left\{b_{i}\right\}$, can be shown to be a distal extension of $\left(X, T^{\prime}\right)$ with $\left(x_{\epsilon}^{*}, x_{-\epsilon}^{*}\right) \in Q$.

In the above example the phase group is nonabelian. We now provide an example in which the phase group is abelian.

ExAMPLE 2 (McMAHON). Let $Z_{2}=\{0,1\}$ be the cyclic group of order 2 $(1+1=0)$. Let $C=\Pi_{-\infty}^{\infty} Z_{2}$ and $T=\amalg_{-\infty}^{\infty}=\{f \in C: f(n)=0$ for all but a finite set of $n$ 's\}. Note $T$ is a dense subgroup of $T$ and letting $T$ act on $C$ by right multiplication makes $(C, T)$ an equicontinuous minimal set.

Let $W=C \times Z_{2}$ and let $f_{n} \in C$ defined by

$$
f_{n}(m)= \begin{cases}0 & \text { if } m=n \\ 1 & \text { if } m \neq n\end{cases}
$$

Define a homeomorphism $\theta_{n}$ on $W$ by 


$$
\theta_{n}(f, z)=\left(f+f_{n}, z+f(n-1)+f(n+1)\right) \quad \text { (addition modulo 2). }
$$

Now note that $\theta_{n}^{2}=$ identity. Also $\theta_{n} \theta_{m}=\theta_{m} \theta_{n}$ since

$$
\begin{aligned}
\theta_{n}\left(\theta_{m}(f, z)\right)= & \theta_{n}\left(f+f_{m}, z+f(m-1)+f(m+1)\right) \\
= & \left(\left(f+f_{m}\right)+f_{n},(z+f(m-1)+f(m+1))\right. \\
& \left.\quad+f(n-1)+f_{m}(n-1)+f(n+1)+f_{m}(n+1)\right)
\end{aligned}
$$

$\left(\right.$ note $\left(f+f_{m}\right)(n-1)=f(n-1)+f_{m}(n-1)(\bmod 2)$, for $\left.f+f_{m} \in C\right)$, and similarly

$$
\begin{aligned}
\theta_{m} \theta_{n}(f, z)=\left(f+f_{n}+f_{m}, z+f(n-1)+f(n+1)\right. & \\
& \left.+f(m-1)+f_{n}(m-1)+f(m+1)+f_{n}(m+1)\right) ;
\end{aligned}
$$

the commutativity follows from the fact that

$$
f_{n}(m-1)=\left\{\begin{array}{lll}
0 & \text { if } & n \neq m-1 \\
1 & \text { if } & n=m-1
\end{array}\right\}=\left\{\begin{array}{lll}
0 & \text { if } & n+1 \neq m \\
1 & \text { if } & n+1=m
\end{array}\right\}=f_{m}(n+1) .
$$

We may define an action of $T$ on $W$ by $w t=\theta_{n_{1}} \circ \cdots \circ \theta_{n_{t}}(w)$ where $\left\{n_{1}, \ldots, n_{t}\right\}$ is the set of integers for which $t(n)=1$ (recall that $T=\{f \in C$ : $f(n)=0$ for all but a finite number of $n$ 's $\}$ ).

The minimality of $(W, T)$ follows from the minimality of $(C, T)$, the openness of the homomorphism $\pi:(W, T) \rightarrow(C, T),(f, z) \mapsto f$, and the fact that for the identity $e$ of $C(e(m)=0$ for every $m) \theta_{n+1} \theta_{n}(e, z) \rightarrow(e, z+1)$ as $n \rightarrow \infty$.

Also $\theta_{n}(e, z) \rightarrow(e, z)$ and so we have $\left(\theta_{n}(e, z),(e, z)\right) \rightarrow((e, z),(e, z))$ while for $f_{n+1} \in T$,

$$
\left(\theta_{n}(e, z),(e, z)\right) f_{n+1}=\left(\theta_{n+1} \theta_{n}(e, z), \theta_{n+1}(e, z)\right) \rightarrow((e, z+1),(e, z)) .
$$

Thus $(e, z)$ is regionally proximal to $(e, z+1)$.

(3.5) Definition. A homomorphism $\phi:(X, T) \rightarrow(Y, T)$ is an $I$-homomorphism if there exist an ordinal $\Lambda$, transformation groups $X_{\gamma}$ for $\gamma \leqslant \Lambda$ and homomorphisms $\phi_{\beta}^{\gamma}: X_{\gamma} \rightarrow X_{\beta}$ for $\beta<\gamma$ such that $X_{\Lambda}=X, X_{0}=Z, \phi_{\beta}^{\gamma}$ are induced by $\phi, \phi_{\gamma}^{\gamma+1}: X_{\gamma+1} \rightarrow X_{\gamma}$ is almost periodic, and $X_{\gamma}=\operatorname{inv} \lim \left\{X_{\beta}: \beta<\gamma\right\}$ if $\gamma$ is a limit ordinal.

Note that $X_{1}$ can be taken to be $X / S(\phi)$ by replacing $X_{\gamma}$ by the homomorphic image of $X$ in $X_{\gamma} \times X / S(\phi)$ for each $\gamma$, under the map $\left(\phi_{\gamma}^{\Lambda}, \pi\right)$, where $\pi$ is the projection of $X$ onto $X / S(\phi)$.

(3.6) REMARK. If $(X, T)$ is a minimal set with metric phase space or if $(X, T)$ is the inverse limit of minimal sets with metric phase space (called quasiseparable), then every distal homomorphism $\phi:(X, T) \rightarrow(Y, T)$ is an I-homomorphism (see 15.4 of [6]). If $T$ is separable, then by 3.3 of $[15],(X, T)$ is 
quasi-separable for every minimal set $(X, T)$ and so every distal homomorphism from a minimal set is an $I$-homomorphism.

(3.7) THEorem. If $(X, T)$ is minimal, $\phi:(X, T) \rightarrow(Y, T)$ an I-homomorphism, and $T \in T$, then the projection of $X$ onto $X / S(\phi)$ has connected fibers.

Proof. Assume $\left(X_{1}, T\right)=(X / S(\phi), T)$ in the definition of $I$-homomorphism. Let $\gamma$ be the first ordinal such that $\phi_{1}^{\gamma}:\left(X_{\gamma}, T\right) \rightarrow\left(X_{1}, T\right)$ does not have connected fibers. Note that $\gamma$ cannot be a limit ordinal since the inverse limit of homomorphisms with connected fibers has connected fibers. Consider

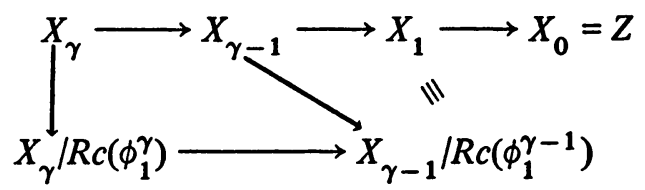

Then $X_{\gamma-1} / R c\left(\phi_{1}^{\gamma-1}\right)$ is isomorphic to $X_{1}=X / S(\phi)$ and $X_{\gamma} / R c\left(\phi_{1}^{\gamma}\right)$ is an inverse limit of finite extensions of $X_{1}$. By 3.1 and 3.2 and the maximality of $X / S(\phi)$ every finite extension is one-to-one and so $X_{\gamma} / R c\left(\phi_{1}^{\gamma}\right)$ is isomorphic to $X_{1}$ and $\phi_{1}^{\gamma}$ has connected fibers.

(3.8) THEOREM. Suppose $\phi$ is a homomorphism from the minimal set $(X, T)$ onto $(Y, T)$ and $T$ is the direct product of a group $H$ with a compact group $K$. Let $\left(X^{*}, T\right)$ be a minimal set in $(X, H)$ and let $\theta$ be the restriction of $\phi$ on $X^{*}$. If $S_{T}(\phi)=R_{T}(\phi)$, then $Q_{T}(\phi) \cap X^{*} \times X^{*}=Q_{H}(\theta)$ (where the subscripts $T$ and $H$ are reminders of what group is acting). $x$ in $X^{*}$.

Proof. We first show that $S(\phi)=R(\phi)$ implies that $R(\phi)(x) \subseteq X^{*}$ for all

By 2.32 of [8], the class of all orbit-closures under $H$ is a star-closed decomposition of $X$; hence the quotient space $Z$ is a compact Hausdorff space. Furthermore since $H$ is normal in $T$, there is a natural action of $T$ on $Z$ and under this action $(Z, T)$ is an almost periodic minimal set. Let $\pi$ be the natural map from $(X, T)$ onto $(Z, T)$ and consider the following diagram

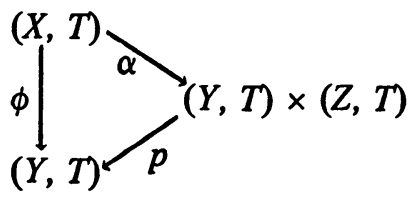

where $\alpha(x)=(\phi(x), \pi(x))$ and $p(y, z)=y$. Since $(Z, T)$ is almost periodic, it is clear that $(\alpha(X), T)$ is an almost periodic extension of $(Y, T)$. The assumption that $S(\phi)=R(\phi)$ implies that there is no nontrivial almost periodic extension through $\phi$. Thus $\left.p\right|_{\alpha(X)}$ must be trivial and $R(\phi)(x) \subseteq X^{*}$ for all $x$ in $X^{*}$.

Since $\left\{X^{*} k: k \in K\right\}$ is a decomposition of $X$ and $R(\phi)(x) \subseteq X^{*}$ for all $x$ in $X^{*}$, we have that for $\left(x_{1}, x_{2}\right) \in R(\phi)$ there exist $k$ in $K$ such that $\left(x_{1} k, x_{2} k\right)$ 
$\in R(\phi) \cap X^{*} \times X^{*}=R(\theta)$. Now suppose $\left(x, x^{\prime}\right) \in Q(\phi) \cap X^{*} \times X^{*}$. Then by the above there exist nets $\left(x_{n}, x_{n}^{\prime}\right)$ in $R(\theta), k_{n}^{*}$ in $K$ and $t_{n}=h_{n} k_{n}$ in $T=H \times K$, such that $\left(x_{n}, x_{n}^{\prime}\right) k_{n}^{*} \rightarrow\left(x, x^{\prime}\right)$ and $\left(x_{n}, x_{n}^{\prime}\right) h_{n} k_{n} \rightarrow(x, x)$. We may assume that the necessary limits exist in the following, otherwise take subnets. Let $k^{*}=$ $\lim k_{n}^{*}$ and $k=\lim k_{n}$. Then $x=\lim x_{n} k_{n}^{*}=\left(\lim x_{n}\right)\left(\lim k_{n}^{*}\right)=\lim \left(x_{n} k^{*}\right)$ and $x^{\prime}=\lim x_{n}^{\prime} k_{n}^{*}=\lim x_{n}^{\prime} k^{*}$. Also $\lim x_{n} h_{n}=\lim x_{n} h_{n} k_{n} k^{-1}=\lim x_{n}^{\prime} h_{n} k_{n} k^{-1}=$ $\lim x_{n}^{\prime} h_{n}$, so $\lim x_{n} k^{*} h_{n}=\lim x_{n} h_{n} k^{*}=\lim x_{n}^{\prime} h_{n} k^{*}=\lim x_{n}^{\prime} k^{*} h_{n}$. We have shown $\left(x, x^{\prime}\right) \in Q_{H}(\theta)$ once we show that $x_{n} k^{*}$ and $x_{n}^{\prime} k^{*}$ are elements of $X^{*}$. Now $x=\lim x_{n} k_{n}^{*}=\left(\lim x_{n}\right) k^{*} \in X^{*} k^{*}$. So $X^{*}=X^{*} k^{*}$ and $x_{n} k^{*}, x_{n}^{\prime} k^{*} \in X^{*}$.

(3.9) REMARK. The same technique could be used to show that if $S_{T}(\phi)=R_{T}(\phi)$ and if the almost periodic points in $(R(\phi), T)$ are dense in $R(\phi)$, then the almost periodic points in $(R(\theta), H)$ are dense in $R(\theta)$.

(3.10) COROLlaRY. If $T$ is the direct product of a separable group $H$ with a compact group $K$, then every distal homomorphism from a minimal set $(X, T)$ onto a minimal set $(Y, T)$ is an I-homomorphism.

Proof. See 4.25 of [5] and 3.3 of [14].

(3.11) COROLLARY. If $T$ is the direct product of a compactly generated separable group with a compact group and if $(X, T)$ is a distal minimal set with totally disconnected phase space, then $(X, T)$ is equicontinuous.

Proof. Note $T \in T$, so the result follows from 3.7 and the above.

(3.12) REMARK. As noted in 6.03 of [8] a generative topological group is isomorphic to $C \times I^{n} \times R^{m}$ where $C$ is a compact abelian group, $I$ is the group of integers, $R$ is the group of reals, and $n, m$ are nonnegative integers. Thus the above is a generalization of previous results [1].

$\$ 4$

We first state some known results that are used in the proof of 4.1. The first is a theorem of Veech, Theorem 3.1 of [12]. Let $\pi:(X, T) \rightarrow(Y, T)$ be a homomorphism of minimal sets and $X$ and $Y$ be metric spaces. There exist canonically determined minimal sets $\left(X^{*}, T\right)$ and $\left(Y^{*}, T\right)$ and homomorphisms $p, q$, and $\pi^{*}$ such that

(i) The diagram

is commutative.

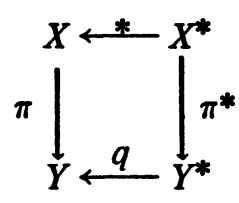

(ii) $p$ and $q$ are almost automorphic homomorphisms (i.e. $p^{-1}(x)$ is a singleton for some $x$ in $X$ ).

(iii) $\pi^{*}$ is an open map. 
In a related result, Theorem 1.2 of [10], the metric assumption is dropped, (ii) is weakened to " $p$ and $q$ are proximal homomorphisms", (iii) is strengthened to " $\pi^{*}$ is open and the almost periodic points in $R\left(\pi^{*}\right)$ are dense in $R\left(\pi^{*}\right)$ ". It is clear from the proofs of the two theorems above that if $\pi^{-1}(y)$ is finite for some $y$ in $Y$, then $\pi^{*-1}\left(y^{*}\right)$ is finite for some $y^{*}$ in $Y^{*}$ and since $\pi^{*}$ is open, $\pi^{*}$ is a finite-to-one almost periodic homomorphism.

(4.1) Proposition. Suppose $(X, T)$ is minimal and $Q\left(x_{0}\right)$ is finite for some $x_{0}$ in $X$. Then $S=Q$ and there exist minimal sets $\left(X^{*}, T\right)$ and $\left(Y^{*}, T\right)$ and homomorphism $\alpha, \beta, \theta^{*}$ such that $\alpha$ and $\beta$ are proximal, $\theta^{*}$ is a finite-to-one almost periodic homomorphism and the following diagram commutes

where $\theta$ is the canonical map.

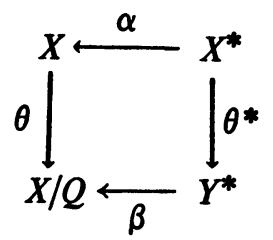

Also if $X$ is metric, then we can take $X^{*}$ to be metric and $\alpha$ and $\beta$ to be almost automorphic homomorphisms.

Proof. All we need to show is that $S=Q$; the rest follows from the above. $Q\left(x_{0}\right)$ finite implies that $x_{0} J$ is finite where $J$ is the set of idempotents in some minimal right ideal of the enveloping semigroup of $(X, T)$. Then $X u=$ $\{x u: x \in X\}$ is dense in $X$ for every $u \in J$ since given any open set $U$ there exists $t \in T$ such that $x_{0} J t \in U$ and if $v \in J$ with $u t^{-1} v=u t^{-1}$, then $v t u=v t$ and $x_{0} v t u \in U$. The fact that $S=Q$ now follows immediately from 2.10 of [10]. As an alternate approach, one could show that $S=Q$ in this case by essentially the same proof as that of 2.13 of [7].

(4.2) Example. The following is an example in which $X \neq X^{*}, Y^{*} \neq X^{*}$, and $Y^{*} \neq X / Q(\phi)$ in the above proposition.

Let $(X, T)$ be a minimal set with $Q_{X}(x)$ finite for some $x$ in $X$ and with an open-closed subset $A$ such that there exist points $x^{\prime} \in A$ and $x^{*} \notin A$ with $\left(x^{\prime}, x^{*}\right) \in P_{X}$. For example, see 5.29 of [6]. Take two copies $X_{-1}, X_{1}$ of $X$ and let $W=X_{-1} \cup X_{1}$ be their topological sum. Define $\sigma: W \rightarrow W$ by

$$
\sigma\left(x_{i}\right)= \begin{cases}x_{i} & \text { if } x_{i} \notin A_{i}(=A \subseteq X), \\ x_{-i} & \text { if } x_{i} \in A_{i}\end{cases}
$$

Let $T$ act on $W$ by $x_{i} t=(x t)_{i}$ and let $T^{*}$ be the group of homeomorphisms of $W$ generated by $\sigma$ and $T$. Then $\left(W, T^{*}\right)$ is a minimal set. If $Q_{X}(x)$ is finite, then $Q_{W}\left(x_{1}\right)$ has at most twice as many points. Also, $\left(x_{-1}^{\prime}, x_{1}^{*}\right) \in P_{W},\left(x_{1}^{\prime}, x_{1}^{*}\right) \in$ $P_{W}$, but $\left(x_{1}^{\prime}, x_{1}^{\prime}\right) \notin P_{W}$. Now $W / Q_{W} \neq Y^{*}$ since if $Y^{*}=W / Q_{W}$, then by the 
proof of 1.2 of [10] and of 3.1 of [12], $W^{*}$ would equal $W$ which is not the case since $\phi: W \rightarrow W / S$ is not distal. Also $W^{*} \neq Y^{*}$ since $W^{*}=Y^{*}$ implies that $\phi$ is proximal and by the above $\phi$ is not proximal.

Finally $W \neq W^{*}$. For if $W=W^{*}$, then $\beta$ proximal implies $\phi^{*}\left(x_{1}^{\prime}\right)$ and $\phi^{*}\left(x_{-1}^{\prime}\right)$ are proximal. So there exists some idempotent $u$ in the enveloping semigroup of $\left(W^{*}, T\right)$ with $\phi^{*}\left(x_{1} u\right)=\phi^{*}\left(x_{1}^{\prime}\right) u=\phi^{*}\left(x_{-1}\right) u=\phi^{*}\left(x_{-1} u\right)$ and so $\phi^{*}$ must identify $X_{1}$ with $X_{-1}$. Then $\phi$ would have to identify $X_{1}$ with $X_{-1}$ which it clearly does not. So $W \neq W^{*}$.

\section{REFERENCES}

1. Robert Ellis, Distal transformation groups, Pacific J. Math. 8 (1958), 401-405. MR 21 \#96.

2. - A semigroup associated with a transformation group, Trans. Amer. Math. Soc. 94 (1960), 272-281. MR 23 \#A961.

3. - The construction of minimal discrete flows, Amer. J. Math. 87 (1965), 564-574. MR 32 \#3053.

4. - Group-like extensions of minimal sets, Trans. Amer. Math. Soc. 127 (1967), 125-135. MR 36 \#4544.

5. The structure of group-like extensions of minimal sets, Trans. Amer. Math. Soc. 134 (1968), 261-287. MR 38 \#6569.

6. Lectures on topological dynamics, Math. Lecture Note Series, Benjamin, New York, 1969. MR 42 \#2463.

7. Robert Ellis and Harvey Keynes, A characterization of the equicontinuous structure relation, Trans. Amer. Math. Soc. 161 (1971), 171-183. MR 43 \#8069.

8. W. H. Gottschalk and G. A. Hedlund, Topological dynamics, Amer. Math. Soc. Colloq. Publ., vol. 36, Amer. Math. Soc., Providence, R. I., 1968.

9. E. Hewitt and K. A. Ross, Abstract harmonic analysis. Vol. I: Structure of topological groups. Integration theory, group representations, Die Grundlehren der math. Wissenschaften, Band 115, Academic Press, New York; Springer-Verlag, Berlin, 1963. MR 28 \#158.

10. D. McMahon and T. S. Wu, On proximal and distal extensions of minimal sets, June 1974, Bulletin of Academia Sinica.

11. R. Sacker and G. Sell, Finite extensions of minimal transformation groups, Trans. Amer. Math. Soc. 190 (1974), 325-334.

12. William A. Veech, Point-distal flows, Amer. J. Math. 92 (1970), 205-242. MR 42 \#2462.

13. - Almost automorphic functions on groups, Amer. J. Math. 87 (1965), 719-751. MR 32 \#4469.

14. - Generalizations of almost periodic functions, Informal notes for lectures at Sir George Williams University, Montreal, Quebec, December 28-January 3, 1971.

15. T.-S. Wu, Homomorphisms in topological dynamics, Hung-ching Chow Sixty-fifth Anniversary Volume, Math. Res. Center Nat. Taiwan Univ., Taipei, 1967, pp. 53-61. MR 37 \#4801.

DEPARTMENT OF MATHEMATICAL SCIENCES, UNIVERSITY OF CINCINNATI, CINCINNATI, OHIO 45224

DEPARTMENT OF MATHEMATICS, CASE WESTERN RESERVE UNIVERSITY, CLEVELAND, OHIO 44106 (Current address of T. S. Wu)

Current address (D. McMahon): Department of Mathematical Sciences, New Mexico State University, Las Cruces, New Mexico 88003 